\title{
Three-Level Decomposition for the Analysis of Turbulent Flow over Rough-Wall
}

\author{
K. Bhaganagar ${ }^{1 \dagger}$ and R. Leighton ${ }^{2}$ \\ ${ }^{I}$ Department of Mechanical Engineering, University of San Antonio, TX, 78248, USA \\ ${ }^{2}$ Ann Arbor, Michigan, USA \\ $\dagger$ Corresponding Author Email:kiran.bhaganagar@utsa.edu
}

(Received August 8, 2010; accepted November 4, 2011)

\begin{abstract}
To improve the understanding of the near-wall region in a rough-wall turbulent boundary layer, we use a three level decomposition as an alternative formulation to the classical Reynolds decomposition. The instantaneous flow variable is now decomposed to a time-space averaged mean flow, a steady mean wake flow around the roughness (i.e. steady but spatially varying motions), and a residual fluctuating flow. In this paper, we present the momentum transport equations for these three components of the decomposition. These transport equations for the three velocity components will facilitate to establish and understand the local interactions of the mean flow, turbulence and wall roughness. We analyze the relative significance of these terms. The fundamental equations are derived within the immersed boundary representation of roughness elements. Total shear stress for rough-wall is obtained from the stress balance equation consisting of stress due to the roughness wake components, the Reynolds stress, the viscous stress and the stress due to the boundary force from the roughness. In order to evaluate the relative contribution of the components in this three-level decomposition, we use direct numerical simulation (DNS) to simulate flow in a channel with rough-walls. Surface roughness has been introduced using immersed boundary methods. The flow simulations are performed at $\mathrm{Re}_{\tau}=180$ and roughness height $h^{+}=5,10,20$ for egg-carton roughness elements.
\end{abstract}

Keywords: Turbulence, Rough-wall, Direct numerical simulation, Three-level decomposition, Roughness wake, Double averaging

\section{NOMENCLATURE}

$\begin{array}{ll}\mathrm{F}_{\mathrm{bu}} & \text { mean boundary drag } \\ F_{b i} & \quad \begin{array}{l}\text { i component of the instantaneous } \\ \text { immersed boundary force }\end{array} \\ h & \begin{array}{l}\text { roughness height } \\ k_{\mathrm{s}}\end{array} \\ \mathrm{n}_{\mathrm{i}} & \text { sand-grain roughness height } \\ u_{\omega} & \text { boundary normal } \\ u^{\prime} & \text { deviation from temporal mean } \\ u^{\prime \prime} & \text { deviation from spatial mean } \\ \underline{\omega} & \text { wall-normal vorticity } \\ u_{i}(x, t) & \text { i component of theinstantaneous velocity } \\ u_{i}^{\omega} & \text { i component of steady wake velocity }\end{array}$

\section{INTRODUCTION}

The near-wall dynamics of turbulent boundary layer with rough-walls is an important ongoing controversial research area. Our basic understanding of the underlying mechanisms and the physics of the roughwall systems rely on the accuracy in both data

\begin{tabular}{|c|c|}
\hline$\hat{U}_{i}(y)$ & horizontal and time averaged mean \\
\hline$x_{b}$ & location of boundary \\
\hline $\begin{array}{l}y_{w} \\
\text { overbar }\end{array}$ & $\begin{array}{l}\text { distance from the wall } \\
\text { time averaged mean }\end{array}$ \\
\hline$<>$ & spatial averaging over constant y planes \\
\hline+ & wall-units \\
\hline$\delta$ & channel half-height \\
\hline$\delta_{t}$ & $\begin{array}{l}\text { distance from wall to } y \text { location to minimum } \\
\text { rms of u velocity }\end{array}$ \\
\hline$\sigma$ & roughness elements \\
\hline$\sigma_{\mathrm{o}}$ & mean offset of immersed boundary \\
\hline
\end{tabular}

measurements as well as in data interpretation. For data measurements, recent efforts have focused on obtaining detailed experimental and simulation database for flow over rough-walls. However, for data interpretation, the traditional analytical methods and the perspectives suitable for smooth-wall turbulence are clearly insufficient for rough-wall turbulence. One of the major 
deficiencies in our understanding of rough-wall turbulence is the lack of inclusion of both turbulence productions due to the roughness elements as well as roughness drag introduced by these elements in the transport equations (Leighton 2009).

The starting point for analyzing the various sources of the turbulence physics are the transport equations for mean, momentum and energy. However, these equations do not contain an explicit information regarding roughness, for example, the time-averaged Navier-Stokes (RANS) equations will not contain the roughness drag term and tells us little about the effects of roughness. Hence, alternative form of transport equations is required which can elucidate the roughness physics.

We present an alternative set of transport equations suitable for rough-walls. The existence of roughness elements complicates the averaging process in this effort. Either the averaging occurs within the strictly liquid domain(Durbin et al. 2001), or it occurs within the combined solid-liquid domain (Raupachet al. 1991; Raupach and Shaw 1982). The former approach is simpler to interpret, but it is harder to generalize and almost meaningless when applied to random roughness. The latter approach is more difficult to interpret, since the averaging includes the zero velocity contributions from within the roughness elements. The advantage of this approach is that the transport equations are general and they explicitly contain roughness contribution such as mean boundary drag and production of turbulence by the roughness. Further, it is shown that the form and viscous drag due to the roughness elements is explicitly included within the mean momentum equations as a result of the spatial averaging process. In multiphase averaging approach, following Drew (1983) an indicator function, $H$ is defined as unity in the fluid phase and zero in the solid phase, is used as a weighting factor in the averaging process. As a consequence of this approach, the roughness contributes to an interfacial source term distributed in space. In this paper, we present the averaging within the combined solid-liquid domain.

A three-level decomposition of the instantaneous flow fields is used to better focus on the underlying near-wall hydrodynamics. The three-level decomposition partitions the flow variables into a space-time average mean component, a time averaged component representing the steady flow around the roughness elements and a residual fluctuating flow. By first averaging in time, then by applying the spatially horizontal averaging approach the time averaged flow is further divided into a spatial mean term (averaged in planes parallel to the boundary) and a steady residual term. The transport equations are derived for the three velocity components. These transport equations for the above three components will facilitate to establish and understand the local interactions of the energy and stress of the mean flow, the turbulence and wall roughness. This will clearly provide the detailed mechanisms. In this study, we will present a framework for the transport of momentum balance.

The paper is arranged as follows: Section 2 deals with the three-level decomposition of the flow fields and the derivation of the transport of the momentum equation based on the flow fields is in Section 3. Section 4 is the details of the DNS tool used to simulate the flow fields. The evaluation of the momentum balance equations are presented in Section 5, and the discussion with conclusions and future work are presented in Section 6.

\section{THREE-LEVEL DECOMPOSITION}

In this section we present the three-level decomposition of the instantaneous velocity into three components. The three-level flow decomposition discussed below is very complementary to approaches presented before by Raupach and Shaw (1982)and Pokrajacet al. (2008) though we will be focusing on applying the approach specific to immersed boundary method for direct numerical simulation (DNS).

We use the classical decomposition of the instantaneous velocity into a long-time mean (represented by an over bar) and a deviation from the time mean (represented by single prime), which we refer to as the time decomposition. The decomposition used by Raupach and Shaw (1982) into a horizontally averaged mean component (represented by angle brackets) and a deviation from this spatial mean (represented by double prime), which we refer to as the spatial decomposition. These averages are explicitly defined as:

$\overline{u_{i}(\underline{x})}=\frac{1}{T} \int_{0}^{T} u_{i} d t$
$\left\langle u_{i}(\underline{x}, t)\right\rangle=\frac{1}{A} \int_{A} u_{i} d A$

where, $\mathrm{T}$ is the averaging time interval and $\mathrm{A}$ is the planar area of the horizontal $(x-z)$ plane. The roughness elements do not complicate the application of the time averaging, but they do complicate the planar averaging. Raupachand Shaw (1982) defined their planar area as excluding the roughness elements and provide an excellent discussion of the impact of that assumption on the development of the transport equations of the mean properties. An alternate approach based on multiphase averaging (Joseph 2002) was used in Leighton and Walker (2007). In that work, an indicator function was used to discriminate the fluid domain from the solid roughness element. Both approaches are constructed to explicitly exclude contributions from the roughness elements to the averages. These two approaches yield equivalent results when applied to the governing equations, including explicit terms related to roughness drag and turbulence production by roughness. The three level decomposition adopted herein exploits the observation that the flow within the roughness elements by virtue of the imposed immersed boundary force is small.

The instantaneous velocity can be decomposed into four terms by the sequential application of the time decomposition (Eq. (1)) and spatial decomposition (Eq. (2)) as follows:

$$
u_{i}=\overline{u_{i}}(\underline{x})+u_{i}^{\prime}(\underline{x, t})
$$

where, $u_{i}(\mathrm{x})$ retains the time averaged wake flow around the roughness elements.

The spatial decomposition based on Eq. (2) is defined as:

$u_{i}(\underline{x}, t)=\left\langle u_{i}(\underline{x}, t)\right\rangle+u_{i}^{\prime \prime}(\underline{x}, t)$ 
where, [-]" represents the spatial variability (i.e. wakes). To keep the notation clean, we introduce spatially- and time-averaged velocity:

$$
\hat{U}_{i}(y)=\left\langle\overline{u_{i}(\underline{x})}\right\rangle=\overline{\left\langle u_{i}(\underline{x}, t)\right\rangle}
$$

also referred to as the double-averaged mean velocity and a mean wake flow $u_{i}^{w}(x)$ defined as

$$
u_{i}^{w}(\underline{x})=\overline{u_{i}(\underline{x}, t)}-\hat{U}_{i}(y)
$$

On performing long-time average of Eq. (4), we obtain,

$$
\overline{u_{i}(\underline{x}, t)}=\left\langle\overline{u_{i}}(\underline{x}, t)\right\rangle+\left[u_{i}(\underline{x})\right]^{\prime}
$$

On substituting Eq. (6) and Eq. (7) in Eq. (3), we obtain

$$
u_{i}(\underline{x}, t)=\hat{U}_{i}(y)+u_{i}^{w}(\underline{x})+u_{i}^{\prime}(\underline{x}, t)
$$

In summary, this is the three-level decomposition of the velocity fields we will employ. Here, $\hat{U}_{i}(y)$ is the time-space averaged mean flow, and the canonical Reynolds decomposition. The planar average of Raupach and Shaw would yield the same result scaled by $\frac{A}{A-\varepsilon}$, where $\varepsilon$ is the planar area within the roughness elements. The second term, $u_{i}^{w}(x)$ is the spatially varying but steady motions around and due to the roughness elements. It should be noted $u^{\prime \prime}(\underline{x, t})$ or

$u_{i}^{w}(x)$ is the steady mean wake flow around the roughness, as $u^{\prime \prime}(\underline{x}, t)$ is the fluctuating wake flow. The last term is the fluctuations from the time mean component. This alternative form of decomposition of flow variables to three components instead of the classical two components is more suitable for roughwalls.

In the next section, we derive and present the transport equations for momentum for the components of the three-level decomposition and stress balance equation for a channel flow.

\section{DeRIVATION OF THE MoMentum Transport Equations For THE COMPONENTS OF THREE-LEVEL DECOMPOSITION}

\subsection{Derivation of the transport equation for time-space averaged mean flow $\left(\hat{U}_{i}(y)\right)$}

We start with the Navier-Stokes momentum equation with a body force representation of the immersed boundary,

$\frac{\partial u_{i}}{\partial t}+u_{j} \frac{\partial u_{i}}{\partial x_{j}}=-\frac{1}{\rho} \frac{\partial P}{\partial x_{i}}+v \Delta u_{i}+f_{i}$

where, $f_{i}$ is the body force due to the immersed boundary. On substituting the three-level decomposition of the velocity fields in the momentum equations (i.e. Eq. (9)), on performing the time-average of the resultant momentum equation, and using $\frac{\overline{\partial Q^{\prime}}}{\partial x_{j}}=0$ yields the momentum equation for the mean component,

$\frac{\partial\left(\hat{U}_{i}(y)+u_{i}^{w}(\underline{x})\right)}{\partial t}+\hat{U}_{j}(y) \frac{\partial}{\partial x_{j}} \hat{U}_{i}(y)+$

$u_{j}^{w}(\underline{x}) \frac{\partial}{\partial x_{j}} \hat{U}_{i}(y)+\hat{U}_{j}(y) \frac{\partial}{\partial x_{j}} u_{i}^{w}(\underline{x})+$

$u_{j}^{w}(\underline{x}) \frac{\partial}{\partial x_{j}} u_{i}^{w}(\underline{x})+$

$\overline{u_{j}^{\prime}(\underline{x}, t) \frac{\partial}{\partial x_{j}} u_{i}^{\prime}(\underline{x}, t)}=-\frac{1}{\rho}\left[\frac{\partial\left(\hat{P}(y)+P^{w}(\underline{x})\right.}{\partial x_{i}}\right]+$

$v \Delta\left(\hat{U}_{i}(y)+u_{i}^{w}(\underline{x})\right)+\left[\hat{f}_{i}(y)+f_{i}^{w}(x)\right]$

On performing the spatial average and using $\left\langle Q_{w}\right\rangle=0$, the resultant equation for the time-space averaged mean flow, $\hat{U}_{i}(y)$ is,

$\frac{\partial}{\partial t} \hat{U}_{i}(y)+\frac{\partial}{\partial x_{j}}\left[U_{i}(y) U_{j}(y)+\right.$

$\left.\left\langle u_{i}^{w}(\underline{x}) u_{j}^{w}(\underline{x})\right\rangle+\overline{\left\langle u_{i}^{\prime}(\underline{x}, t) u_{j}^{\prime}(\underline{x}, t)\right\rangle}\right]=$

$\left.-\frac{1}{\rho} \frac{\partial \hat{P}(y)}{\partial x i}+v \Delta \hat{U}_{i}(y)+\hat{f}_{i}(\underline{x}, t)\right)$

Eq. (11) represents the momentum transport equation for time-space averaged mean velocity component.

\subsection{Derivation of transport equation for the fluctuating component $\left(u_{i}^{\prime}\right)$}

The transport equation for $u_{i}^{\prime}(\underline{x}, t)$, the fluctuation from the time-mean is obtained as follows: On using the time decomposition in the Navier- Stokes Equations, Eq. (9), and by performing a time-average on the resultant equation (which is the conventional Reynolds averaged Navier- Stokes equations yields,

$$
\begin{aligned}
& \frac{\partial\left(\bar{u}_{i}(\underline{x})+u_{i}^{\prime}(\underline{x}, t)\right)}{\partial t}+\left(\overline{u_{j}}(\underline{x})+u_{j}^{\prime}(\underline{x}, t)\right) \frac{\partial\left(\bar{u}_{i}(\underline{x})+u_{i}^{\prime}(\underline{x}, t)\right)}{\partial x_{j}}= \\
& -\frac{1}{\rho}\left[\frac{\partial\left(\overline{P(\underline{x})}+P^{\prime}(\underline{x}, t)\right.}{\partial x i}\right]+v \Delta\left(\overline{u_{i}}(\underline{x})+u_{i}^{\prime}(\underline{x}, t)\right) \\
& +\left[\overline{f_{i}(\underline{x})}+f_{i}^{\prime}(\underline{x}, t)\right]
\end{aligned}
$$

On subtracting Eq. (12) from the conventional Reynolds averaged Navier- Stokes equations; we obtain the equation for the unsteady component,

$$
\begin{aligned}
& \frac{\partial u_{i}^{\prime}(\underline{x}, t)_{i}}{\partial t}+\bar{u}_{j}(\underline{x}) \frac{\partial u_{i}^{\prime}(\underline{x}, t)}{\partial x_{j}}+ \\
& u_{j}^{\prime}(\underline{x}, t) \frac{\partial}{\partial x_{j}} \bar{u}_{i}(\underline{x})=-\frac{1}{\rho} \frac{\partial P^{\prime}(\underline{x}, t)}{\partial x_{i}}+ \\
& v \Delta u_{i}(\underline{x}, t)+f_{i}^{\prime}(\underline{x}, t)
\end{aligned}
$$


It should be noted that both the time-average and spatial-average mean of this equation is zero.

\subsection{Derivation of transport equation for the mean wake component $\left(u_{i}^{w}\right)$}

Starting with the time-averaged Navier-Stokes equations, expressing the time averaged mean in terms of the spatial mean (Eq. (7)), by performing a spatial averaging and using $\left\langle Q_{w}\right\rangle=0$, one obtains,

$$
\begin{aligned}
& \frac{\partial\left(\hat{U}_{i}(y)\right)}{\partial t}+\left(\hat{U}_{j}(y)\right) \frac{\partial\left(\hat{U}_{i}(y)\right)}{\partial x_{j}}+\frac{\partial}{\partial x_{j}}\left\langle u_{i}^{w}(\underline{x}) u_{j}^{w}(\underline{x})\right\rangle= \\
& -\frac{1}{\rho} \frac{\partial(\hat{P}(y))}{\partial x i}+v \Delta\left(\hat{U}_{i}(y)\right)+f_{i}(y)
\end{aligned}
$$

On subtracting Eq. (14) from space-averaged NavierStokes equations, we obtain the equation for the wake component as,

$$
\begin{aligned}
& \frac{\partial\left(u_{i}^{w}(\underline{x})\right)}{\partial t}+u_{j}^{w}(\underline{x}) \frac{\partial}{\partial x_{j}} \hat{U}_{i}(y)+\hat{U}_{j}(y) \frac{\partial}{\partial x_{j}} u_{j}^{w}(\underline{x})+ \\
& \left.\frac{\partial}{\partial x_{j}}\left[\overline{\left(u_{i}^{\prime}(\underline{x}, t) u_{j}^{\prime}(\underline{x}, t)\right.}\right)-\left\langle\overline{u_{i}^{\prime}(\underline{x}, t) u_{j}^{\prime}(\underline{x}, t)}\right\rangle\right]+ \\
& \frac{\partial}{\partial x_{j}}\left[u_{i}^{w}(x) u_{j}^{w}(x)-\left\langle u_{i}^{w}(x) u_{j}^{w}(x)\right\rangle\right]= \\
& -\frac{1}{\rho} \frac{\partial\left(P^{w}(\underline{x})\right.}{\partial x i}+v \Delta\left(u_{i}^{w}(\underline{x})\right)+F_{i}^{w}(x)
\end{aligned}
$$

It should be noted that this equation is independent of time, and also its spatial average is zero. To summarize, Eq. (11), Eq. (13) and Eq. (15)represent the transport equations for the three components of the three-level decomposition introduced.

\subsection{Stress balance for a channel flow: Total shear stress for rough-wall}

We derive the stress balance equation for channel flow. We start with the transport equation for the time-space averaged mean $\hat{U}_{i}(y)$ (Eq. (11)). For channel flow, the $\mathrm{x}$ momentum equation simplifies to,

$$
\begin{aligned}
& \frac{\partial\left[-\left\langle u_{2}^{w}(\underline{x}) u_{1}^{w}(\underline{x})\right\rangle-\overline{\left\langle u_{2}^{\prime}(\underline{x}, t) u_{1}^{\prime}(\underline{x}, t)\right\rangle}\right]}{\partial y}= \\
& -\frac{1}{\rho} \frac{\partial P(y)}{\partial y}+v \frac{\partial^{2} \hat{U}(y)}{\partial y^{2}}+\int f_{1} d y
\end{aligned}
$$

On integrating the above equation in the wall-normal direction we obtain the stress balance equation,

$$
\begin{aligned}
& -\left\langle\left\langle u_{1}^{w}(\underline{x}) u_{2}^{w}(\underline{x})\right\rangle+\left\langle\overline{u_{1}^{\prime}(\underline{x}, t) u_{2}^{\prime}(\underline{x}, t)}\right\rangle\right]+ \\
& v\left[\frac{\partial U(y)}{\partial y}\right]+\int\left\langle f_{1}(y)\right\rangle d y=\left(1-\frac{y}{\delta}\right)
\end{aligned}
$$

Here, $\delta$ is the half-height of the channel. On the left hand side of the equation is the total shear for the rough-wall. On the left hand side, the first term represents the stress due to the roughness wake, the second term is the Reynolds stress term, the third term is the viscous stress term, and the last term is the averaged body-force term due to the immersed boundary or the roughness drag term.

\section{Direct Numerical Simulation SOLVER}

Direct numerical simulation solver (Bhaganagar,Kim and Coleman, 2004) to simulate steady flow in a channel with rough walls is used. The DNS solver developed is an accurate, robust, parallel numerical tool to simulate turbulent flow over a complex boundary while retaining the simplicity and efficiency of computation in a Cartesian system by solving the governing Navier-Stokes equations exactly without any approximations. The coordinate system is Cartesian system with $(\mathrm{x}, \mathrm{y}, \mathrm{z})$ being the stream wise, wall-normal and span wise coordinates. The corresponding velocity and vorticity components are represented by $(u, v, w)$ and $\left(\omega_{\mathrm{x}}, \omega_{\mathrm{y}}, \omega_{\mathrm{z}}\right)$ respectively.

The governing equations are expressed in wall-normal velocity- wall-normal vorticity formulation (Bhaganagar, Rempfer and Lumley, 2002). The wallnormal component of the curl of the Navier Stokes equations results in an evolution equation for the wall normal vorticity, $\omega_{y}$. Application of the Laplace operator to the momentum equation for the wall-normal component of velocity yields an equation for that component through the use of the momentum and the pressure Poisson equations. For Reynolds number Re, the resulting equations are a second order evolution equation for wall-normal vorticity, $\omega_{y}$, and a fourth order evolution equation for wall-normal velocity, $\mathrm{v}$ as follows,

$$
\begin{aligned}
& \frac{\partial \omega_{y}}{\partial t}=H_{\omega}+\frac{1}{\operatorname{Re}} \nabla^{2} \omega_{y}, \\
& \frac{\partial \nabla^{2} v}{\partial t}=H_{v}+\frac{1}{\operatorname{Re}} \nabla^{4} v, \\
& v( \pm 1)=\frac{\partial v}{\partial y}( \pm 1)=0,
\end{aligned}
$$

$\omega_{y}( \pm 1)=0$,

where,

$$
\begin{aligned}
& H_{v}=-\frac{\partial}{\partial y}\left[\frac{\partial H_{1}}{\partial x}+\frac{\partial H_{2}}{\partial z}\right]+\left[\frac{\partial^{2}}{\partial x^{2}}+\frac{\partial^{2}}{\partial z^{2}}\right] H_{3} \\
& H_{\omega}=\left(\frac{\partial H_{3}}{\partial x}+\frac{\partial H_{1}}{\partial z}\right]
\end{aligned}
$$

$H=\left(H_{1}, H_{2}, H_{3}\right)=u \times \omega$,

$\Delta=\left[\frac{\partial^{2}}{\partial x^{2}}+\frac{\partial^{2}}{\partial y^{2}}+\frac{\partial^{2}}{\partial z^{2}}\right]$

In the horizontal $x-z$ directions the spatial discretization is done using Fourier series expansion assuming periodicity as the flow is statistically 
homogeneous. In the wall-normal $y$ direction the flow is inhomogeneous, and the flows need to be well resolved. Hence high-resolution compact finite differences have been used to obtain the spatial derivatives. To avoid stringent time step restrictions, a semi-implicit time integration has been implemented to integrate the resultant discretized equations. For the nonlinear terms, an explicit low-storage, three-stage, fourth order Runge- Kutta scheme has been used and for the linear viscous terms, an implicit Crank-Nicholson scheme has been used. The solution at the end of each time-step is the sum of the solution of the explicit part and the implicit part. Refer to Carpenter (Carpenter and Kennedy 1994) for the details of the formulation

\subsection{Immersed boundary method}

The virtual roughness boundary is prescribed within the channel via a body force term, using the immersed boundary method, by enforcing the no-slip condition at this virtual boundary (Yusof 1997). Three-dimensional roughness-elements are introduced with an "egg carton"-shaped surface $\sigma(\mathrm{x}, \mathrm{z})$ such that

$$
\begin{aligned}
& \sigma(x, z)=\sigma_{o}+ \\
& \frac{1}{4} h\left[\begin{array}{l}
-1+\left(1+\sin \left(\frac{2 \pi x}{l x}+\frac{2 \pi y}{l z}\right)\right) \\
\left(1+\sin \left(\frac{2 \pi x}{l x}-\frac{2 \pi y}{l z}\right)\right)
\end{array}\right],
\end{aligned}
$$

where, $\sigma(\mathrm{x}, \mathrm{z})$ is measured with respect to the channel coordinates, in units of $\delta$ (channel half-height), $\mathrm{h}$ is the (peak to valley) roughness height, $\sigma_{o}$ defines the mean offset of the immersed boundary, and $l_{\mathrm{x}}$ and $\mathrm{l}_{\mathrm{z}}$ are the stream wise and span wise wavelengths (peak-to-peak distance) of the roughness elements.

We chose $\sigma_{0}=-0.96$ for all simulations presented here, which prescribes the virtual no-slip roughness surface at the bottom of the DNS domain, just above the lower wall. For this surface, the roughness "bumps" extend $3 \mathrm{~h} / 4$ above $\sigma_{0}$, while the valleys lie $\mathrm{h} / 4$ below it. The definition of the body force term is described as follows: A first order temporal discretization of the Navier-Stokes equations is employed,

$$
\frac{u^{n+1}-u^{n}}{\delta t}+u^{n} \bullet \nabla u^{n}=
$$

$-\nabla p^{n}+\nu \nabla^{2} u^{n}+f$

where $f=(f 1, f 2, f 3)$ is the body force, $u=(u, v, w)$ the velocity vector, $\mathrm{p}$ the pressure, $v$ the kinematic viscosity, $\delta \mathrm{t}$ the time-step increment, and the superscripts $n$ and $n+1$ respectively indicate the current and next time level. On the immersed boundary $\sigma(\mathrm{x}, \mathrm{z})$, the velocity is zero, such that

$u^{n+1}=(0,0,0)$

and the body force is approximated as:

$f=\frac{V-u^{n}}{\delta t}+u^{n} \bullet \nabla u^{n}+\nabla p^{n}-v \nabla^{2} u^{n}$,

where, $\mathrm{V}=(0,0,0)$.

This method gives flexibility in choosing the immersed boundary not found in some other methods, since there is no need to line up the boundary with a grid.

\subsection{Computation of $u_{\tau}$}

The wall shear velocity (friction velocity), $u$ is obtained by solving the $\mathrm{x}$ (stream wise) momentum equation for the mean velocity,

$0=-\frac{d p}{d x}+\frac{1}{\operatorname{Re}} \frac{d^{2} U}{d y^{2}}-\frac{d}{d y} \overline{u v}+f_{1}$

where, $f_{I}$ is the $\mathrm{x}$ component of the body force term $\mathrm{f}$ (refer to Eq. (26)).

As the non-dimensional domain extends from $y=-1$ to $y=+1$ in the wall normal direction, integrating the above equation from the lower wall $(\mathrm{y}=-1)$ to the upper wall $(y=+1)$ results in,

$$
\left(u_{\tau}^{r}\right)^{2}=-2 \frac{d p}{d x}+\left.\frac{1}{\operatorname{Re}} \frac{d U}{d y}\right|_{y=1}+\int_{y=-1}^{y=1} f_{1} d y
$$

where, the stress at the lower rough wall has been defined as $\left(u^{r}\right)^{2}$. The last term of the above equation is evaluated using a trapezoidal rule for a non-uniform mesh. For the smooth (flat) upper wall, the friction velocity is expressed as,

$$
\left(u_{\tau}^{s}\right)^{2}=\left.\frac{1}{\operatorname{Re}} \frac{d U}{d y}\right|_{y=1}
$$

where, the stress at the upper smooth wall has been defined as $\left(u_{s}^{r}\right)^{2} . u$ is computed at every $x-y$ grid point is averaged over all these locations as well as in time (as the statistics have reached a statistically steady state $u$ does not change significantly with time).

\section{Evaluation OF Momentum Balance Equations Using Direct NUMERICAL SIMULATIONS}

We analyze simulations of turbulent flow over roughwall with $3 \mathrm{D}$ roughness elements. The shape of the roughness elements is expressed by Eq. (25). We specify roughness elements with peak-to-peak spacing in the stream wise and span wise directions of 100 wall units in terms of smooth-wall $u$ and three different heights of $h^{+}=5,10$ and 20 .

The three rough-wall cases correspond respectively to equivalent sand-grain roughness of $k_{s}^{+}=10,20$, and 48 ; the ratio of $k_{\mathrm{s}}$ to the physical peak-to-valley height $\mathrm{h}$ for the three cases is thus $1.87,1.85$ and 2. 2 .

These $\mathrm{ks} / \mathrm{h}$ values are analogous to, for example, Case 11 of Schlichting's regular roughness patterns. Once the velocity field reached a statistically steady state, the computations were continued in time for about 30 nondimensionalized units (using $u$ of smooth wall and $\mathrm{H}$ ) to obtain mean statistics (which were gathered by averaging over $\mathrm{x}$ and $\mathrm{z}$ directions as well as time). Tests were performed to verify adequate convergence of the mean results

We begin the analysis by examining the root-meansquare (rms) velocity fluctuations normalized by $u$ 
from the smooth wall. Figure 1 shows the rms of (a) stream wise, (b) wall-normal, and (c) span wise velocity fluctuations for 3 different roughness heights $h^{+}=5.4$, 10.6, 21.6. The fluctuations become more intense with increasing roughness height. Moreover, the peak location moves further away from the wall with

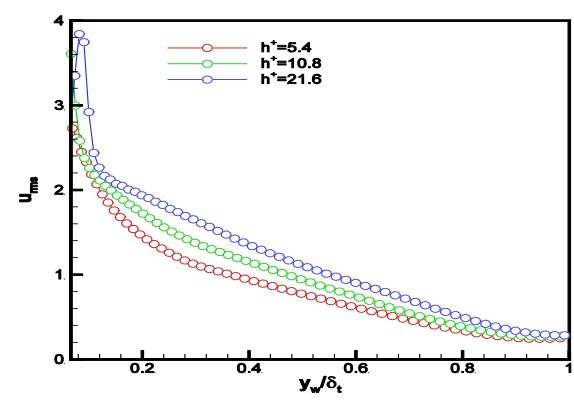

(a) increasing roughness. The distance from the wall $\mathrm{y}_{\mathrm{w}}$ is normalized by ${ }_{t}$, which is defined as the distance from the wall to the y location corresponding to minimum rms velocity fluctuations.

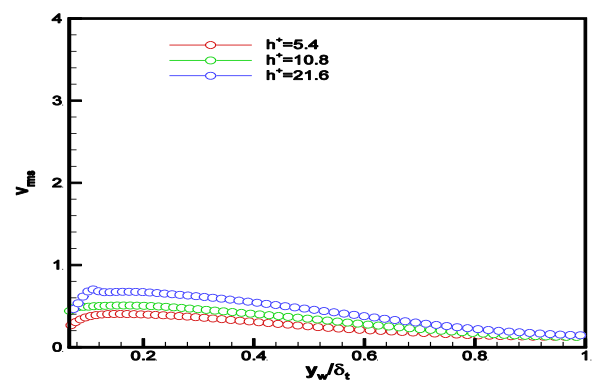

(b)

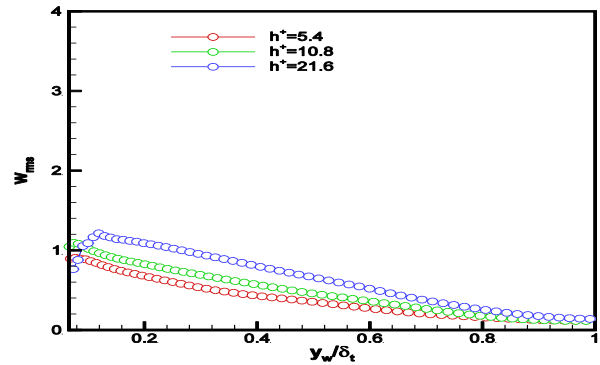

(c)

Fig. 1. (a) Rms of streamwise velocity fluctuations, (b) Rms of wall-normal velocity fluctuations, (c) Rms of spanwise velocity fluctuations plotted vs. wall normal distance

Next, we examine the rms of wake velocity as seen in Fig. 2. It is plotted against the wall distance scaled by $\delta_{t}$.

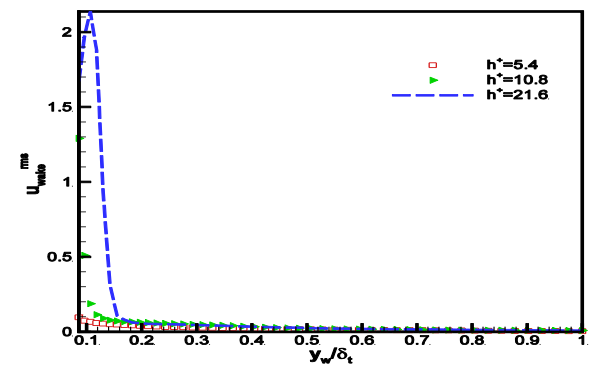

(a)

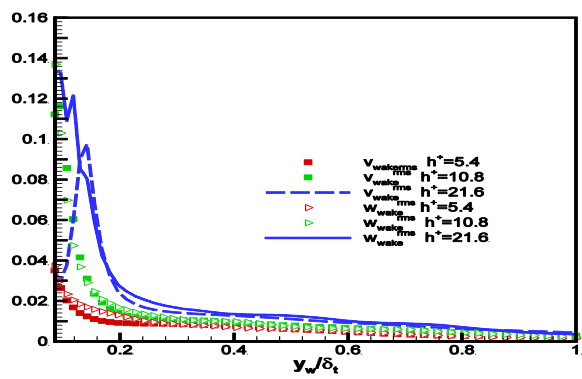

(b)

Fig. 2. rms of the velocity fluctuations rms of wake component (a) streamwise velocity fluctuations scaled by rough-wall $u_{\tau}$, (b) wall-normal and spanwise velocity fluctuations plotted vs wall normal distance.
Figure 2(a) corresponds to the rms of the streamwise component of the wake velocity. The strength of the wake as well as the wall-normal extent of the wake increases with increasing roughness height. The nonzero wake extends up to $y / \delta_{t}=0.46,0.62,0.72$ for $\mathrm{h}^{+}=$ $5.4,10.8,21.6$ respectively. Figure 2(b) shows the rms of the wall-normal and the spanwise components of the wake velocity respectively. It is interesting that though the rms of the $\mathrm{v}$ and $\mathrm{w}$ velocity fluctuations show differences only very close to the wall, but have similar pattern and trend for the rest of the layer. The planar fields of the wake velocity are examined next.

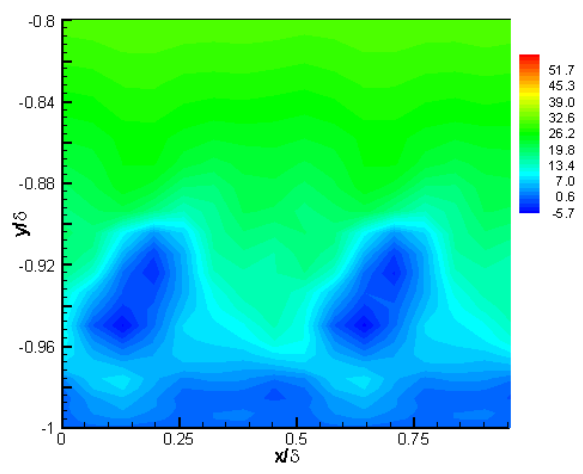

Fig. 3. Wake component of the streamwise velocity in the $x-y$ plane.

Figure 3 shows the streamwise component of the wake velocity in $\mathrm{x}-\mathrm{y}$ plane for $h^{+}=21.6$. Distinct repeated 
patterns of the wake are evident and extend to height of $\mathrm{y} / \delta=-0.92$.

Figure 4(a) shows the wall-normal component of the wake velocity in the $x-y$ plane. Alternate patterns of positive and negative velocity are seen. The structures are more elongated in the wall-normal direction. These structures extend to $y / \delta=-0.66$, which is larger in extent compared to streamwise component. Figure 4(b) shows the spanwise component of the wake; alternate patterns of positive and negative velocity are seen. Unlike the wall-normal components, these structures are more inclined to the horizontal. These plots show distinct patterns of the wake due to the roughness, which is the characteristic feature of the nature of the roughness elements.

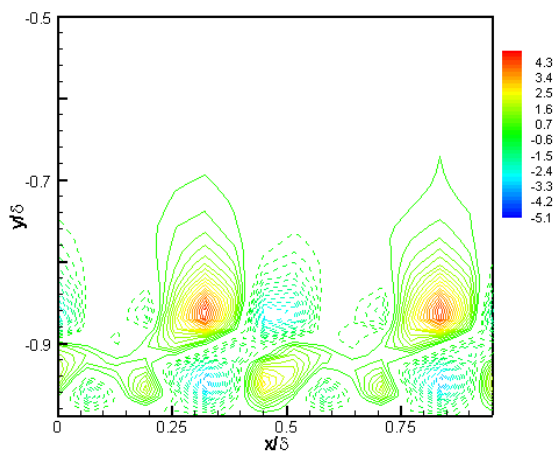

(a)

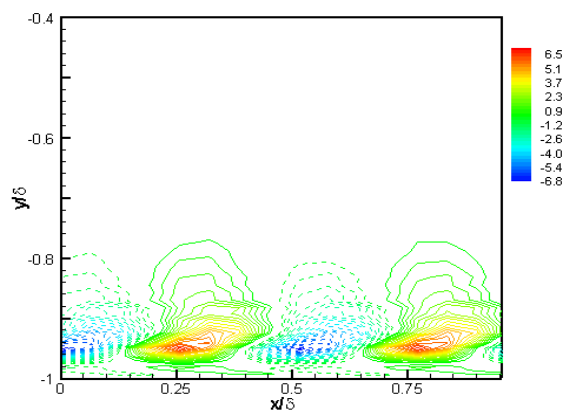

(b)

Fig. 4. (a) Wake component of wall-normal velocity. (b) Wake component of spanwise velocity in $x-y$ plane

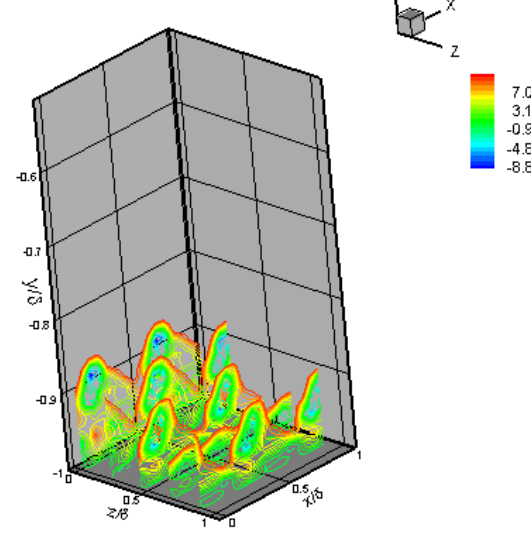

Fig. 5. Wake component of the streamwise velocity in $x-y$ plane at different $z$ locations.
Figure 5 shows the streamwise component of the wake at different $\mathrm{z}$ locations in $x-y$ plane. The staggered nature of the wake, which resembles the pattern of the egg-carton roughness of the wall, is observed.

Figures 6(a) and 6(b) show the rms of the streamwise $\left(f_{x}^{r m s}\right)$ and wall-normal $\left(f_{y}^{r m s}\right)$ components of the body-force respectively plotted in wall-units.

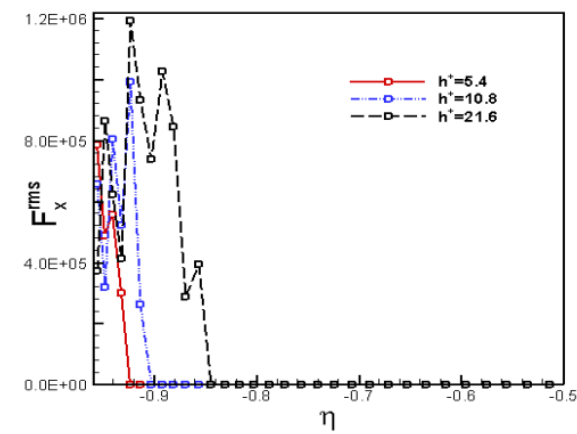

(a)

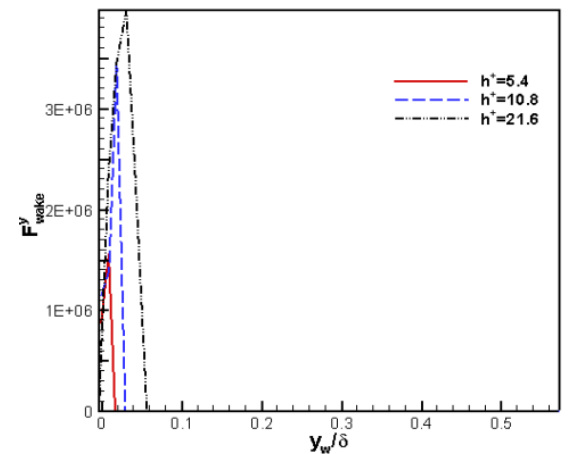

(b)

Fig. 6. rms of body force (a) streamwise velocity fluctuations scaled by rough-wall $\mathrm{u}_{\tau}$, (b) wall-normal velocity fluctuations plotted vs. wall normal.

The streamwise and wall-normal components of the wake portion of the body-force plotted in wall-units are presented in Figs. 7(a) and 7(b), respectively. The strength of the wake increases with increasing roughness height. The location of the peak magnitude is at the same wall-normal distance from the wall. The rms of the body-force and the wake component extend to more or less the same distance from the wall. The rms of the body force and the wake component of the body force is especially useful when comparing roughness of different geometries.

The evaluated stress balance (Eq. (17)) is presented in Fig. 8. The stress is plotted as a function of $y R w$ scaled by channel half-height $\delta$., which is the distance from the rough-wall taking the virtual origin into account. As seen from Eq. (17) the total shear stress at the rough wall consists of the stress due to roughness wake, viscous stress, Reynolds stress and stress due to the body force. This is validated by the linear profile of the total stress from Fig. 8. The stress due to wake components (diamond symbols in Fig.8) extends to $y_{w}^{R}=0.6$, which is well into the outer layer of the turbulent boundary layer, on the other hand, the stress due body force (which is distributed stress due to 
roughness boundary) extends to $y_{w}^{R}=0.1$, which is closer to the wall.

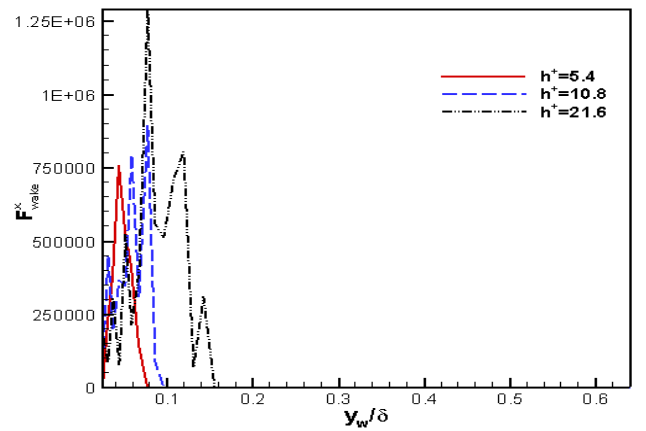

(a)

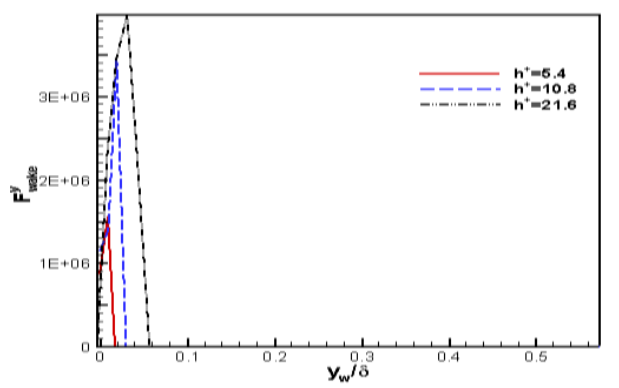

(b)

Fig. 7. rms of wake component of the body force (a) streamwise component scaled by rough-wall $u_{\tau}$, (b) wall-normal component plotted vs. wall normal.

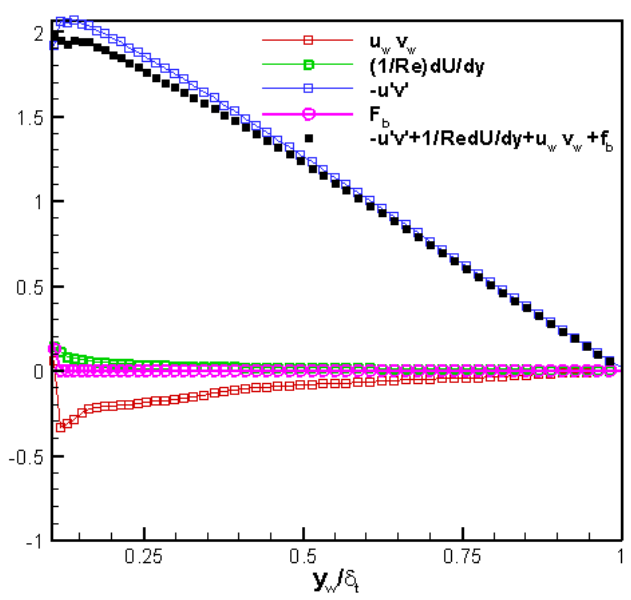

Fig. 8. The stress partition at the wall including roughness stress due to the wake $\left(\left\langle u_{w} v_{w}\right\rangle\right)$, the viscous stress $\left(\frac{1}{\operatorname{Re}} \frac{d U}{d y}\right)$, Reynolds stress $\left.\left(<u^{\prime} v^{\prime}\right\rangle\right)$, stress due to boundary force $\left(F_{r}\right)$ and total stress (sum of all the stresses).

\section{Discussion ANd Conclusions}

The starting point for analyzing the various sources of the turbulence physics are the transport equations for the mean and fluctuating components. However, when these equations are used for the analysis of flow over rough-wall, they do not contain explicit information regarding the effects of roughness. For this purpose, we require an alternative set of transport equations. We introduce the three level decompositions with focus on the immersed boundary method for direct numerical simulations.

This decomposition accounts for the spatial variability due to the roughness. This paper focuses on developing novel set of transport equations for the study of roughwall turbulent boundary layer. The traditional Reynolds decomposition of flow variables has been modified to three-level decomposition consisting of three components. The first component in the three-level decomposition is the time-space averaged mean flow. The second component is spatially varying but steady wake motion due to and around the roughness elements. The third component is the residual fluctuating component from the time-averaged mean.

A reasonable question at this point is why so much effort. The utility of this type of decomposition in conjunction representations of the roughness drag is as a means of examining the interactions of the hydrodynamics and roughness. In particular, the transport equations for each of these components reveal information regarding the mechanisms and the transfer between these components. This approach is unique in that the analysis guided by the balance equations, will focus on the forces and mechanisms of rough-wall turbulence.

We derive the transport equations for the three components of the three level decomposition, namely the time-space averaged mean $(\hat{U}(y))$, the steady wake component $\left(u^{w}(\underline{x})\right)$, and the fluctuating component $\left(u_{i}^{\prime}\right)$. In the transport equation for timespace averaged mean component (Eq. (11)), the left hand of this equation are terms that are analogous to convection terms. The term $\frac{\partial}{\partial x_{j}} \hat{U}_{i}(y) \hat{U}_{j}(y)$ represents the convection of time-space averaged mean momentum. The next term $\frac{\partial}{\partial x_{j}} u_{i}^{w}(\underline{x}) u_{j}^{w}(\underline{x})$ represents the mean transport of the wake momentum by the timespace averaged mean wake component, and finally $\frac{\partial}{\partial x_{j}}<u_{i}^{\prime}(\underline{x}, t) u_{j}^{\prime}(\underline{x}, t)>$ is the mean transport of spatially averaged momentum by the turbulent fluctuations. It is important to recognize that only when $u_{i}^{w}$ and $u_{j}^{w}$ are correlated, there is an additional transfer of momentum due to the wake component. An additional amount of momentum of the mean flow is altered due to the exchange of momentum between the mean flow and the wake. We can further explain these terms by considering the transport equation for the wake (Eq. (15)), it is seen that equal and opposite $\frac{\partial}{\partial x_{j}} u^{w}{ }_{i}(\underline{x}) u_{j}^{w}(\underline{x})$ and $\frac{\partial}{\partial x_{j}}<u_{i}^{\prime}(\underline{x}, t) u_{j}^{\prime}(\underline{x}, t)>$ terms appear. Thus, the wake due to the roughness elements plays an important role in the alteration of the momentum of the mean flow. On exploring further, the mean transport equation (Eq. (11)), the right-hand side 
term $\hat{f}_{i}$ denotes the mean boundary drag. It defines the distribution of roughness drag as double-averaged hydrodynamic stress acting on the boundary, i.e. it represent the mean force acting on the fluid due to the no-slip condition on the boundary. Finally, we use the mean momentum equation to obtain the total stress balance equation for the rough-wall. The total stress due to the rough-wall is due to contribution from the roughness drag (body force), roughness wake, viscous stress and the Reynolds stress.

We simulate the flow in channel with rough-walls covered with "egg-carton" roughness elements using DNS. The simulations are performed for three different roughness heights corresponding to $\mathrm{h}^{+}=5,10,21$. We evaluate the various terms of the momentum transport equations to understand the role of height of the roughness elements. On examining the rms of the velocity fluctuations, the fluctuations become intense with increasing roughness height, and the peak location moves away from the wall. The rms of the wake component of velocity reveals the strength of the wake as well as the wall-normal extent of the wake increases with increasing roughness height. Distinct patterns of wake are revealed on analysis of the $\mathrm{x}-\mathrm{y}$ fields of the streamwise, wall-normal and spanwise. The effect of roughness geometry on the flow is quite evident on the wake structures.

In future, we will present the balance equations for turbulent kinetic energy and turbulent stress equations for the mean, wake and turbulent fluctuating components. Further, we will apply this analytical framework for range of different roughness shapes and thus we will be able to parameterize roughness drag and roughness turbulence production based on the unique set of transport equations specific for rough-wall.

\section{ACKNOWLEDGEMENTS}

The first author would like to acknowledge the financial support from University of Texas Collaborative Initiative Grant 2010-2011

\section{REFERENCES}

Bhaganagar, K., Rempfer, D. and Lumley, J.L. (2002). Direct Numerical Simulation of Spatial Transition to Turbulence using Fourth-Order Vertical Velocity Second-Order Vertical Vorticity Formulation, Journal of Computational Physics, 180(1), 200-228.

Bhaganagar, K., Kim, J., and Coleman, G. (2004). Effect of roughness on wall bounded turbulence, Flow, Turbulence and Combustion, 72(2-4), 463492.
Bhaganagar, K., Coleman, G. and Kim. J. (2007). Effect of roughness on pressure statistics, Physics of Fluids, 19(2), 028103.

Carpenter, M. H., Kennedy, C. A. (1994). A fourth order $2 \mathrm{~N}$-storage Runge-Kutta scheme. NASA TM, 109112.

Drew, D.A. (1983). Mathematical Modeling of TwoPhase Flow, Annual Review of Fluid Mechanics, 15, 261-291.

Durbin, P.A., (2001). Rough wall modification of twolayer k-e, ASME Trans.

Joseph, D.D. (2002). Interrogations of Direct Numerical Simulation of Solid-Liquid Flows .http://efluids.com/efluids/books/Interog-1.pdf.

Leighton, R. I. and Bhaganagar, K. (2010).Turbulence production by rough boundaries. Paper USNCTAM2010-1173, 16th US National Congress of Theoretical and Applied Mechanics, Pennsylvania, State College, USA.

Leighton, R.I., (2009). Turbulence Production by Rough Boundaries, AIAA paper 2009-1609.

Leighton, R.I. and Walker, D. T., (2007). Reynolds Stress Modeling for Rough Wall Boundary Layers, AIAA paper 2007-4615.

Pokrajac, D., Mc Ewan, I. and Nikora, V. (2008). Spatially averaged turbulent stress and its partitioning. Experiment ins Fluids, 45(1), 73-83.

Raupach, M. R., Antonia, R. A., and Rajagopalan, S. (1991). Rough-wall turbulent boundary layers. Appl. Mechanics. Rev., 44(1), 1-20.

Raupach, M.R., Shaw, R.H. (1982). Averaging procedurs for flow within vegetation canopies, Boundary Layer Meteorology, 22(1), 79-90.

Schlichting,H. (1979). Boundary Layer Theory. Seventh edition.

Yusof, J.M. (1997). Combined immersed-boundary/Bspline methods for simulations of flow in complex geometries. CTR-Annual Research Briefs - Stanford Univ./NASA Ames. 